\title{
First-principles study on atomic configuration of electron-beam irradiated $\mathrm{C}_{60}$ film
}

\author{
Tomoya Ono \\ Graduate School of Engineering, Osaka University, Suita, Osaka 565-0871, Japan
}

Shigeru Tsukamoto

Peter Grünberg Institut \& Institute for Advanced Simulation, Forschungszentrum Jülich and JARA, D-52425 Jülich, Germany

(Received 10 May 2011; revised manuscript received 4 July 2011; published 7 October 2011)

\begin{abstract}
Density functional calculations of the atomic configuration of electron-beam irradiated $\mathrm{C}_{60}$ thin films were implemented. By examining the electronic structure and electron-transport properties of $\mathrm{C}_{60}$ clusters, we found that a rhombohedral $\mathrm{C}_{60}$ polymer with $s p^{3}$-bonded dumbbell-shaped connections at the molecule junction is a semiconductor with a narrow band gap. In addition, the polymer changes to exhibit metallic behavior by forming $s p^{2}$-bonded peanut-shaped connections. Conductance below the Fermi level increases and the peak of the conductance spectrum arising from the $t_{u 1}$ states of the $\mathrm{C}_{60}$ molecule becomes obscure after the connections are rearranged. The present rhombohedral polymer, including the $[2+2]$ four-membered rings and peanut-shaped connections, is a candidate for representing the structure of the metallic $\mathrm{C}_{60}$ polymer at the initial stage of electron-beam irradiation.
\end{abstract}

DOI: 10.1103/PhysRevB.84.165410

\section{INTRODUCTION}

Intermolecular electron transport has attracted a great deal of attention in numerous fields of research, accompanied by progress in nanostructure-fabrication technology. Fullerene-based molecular crystals and films are of particular interest because of their very rich physical properties, such as superconductivity and magnetism. The polymerization of $\mathrm{C}_{60}$ molecules in the solid phase using various techniques, e.g., photoirradiation or electron-beam (EB) irradiation, have resulted in new forms of carbon materials. ${ }^{1}$ A first-principles study by Okada and Oshiyama reported that the band gap of the rhombohedral phase of the $\mathrm{C}_{60}$ polymer linked by $[2+2]$ four-membered rings, in which parallel double bonds of adjacent $\mathrm{C}_{60}$ molecules are broken and then single bonds between the $\mathrm{C}_{60}$ molecules are formed (e.g., see Fig. 1), is significantly smaller than that of $\mathrm{C}_{60}$ bulk. $^{2}$ Onoe et al. found that $\mathrm{C}_{60}$ films change to polymers accompanying the insulator-metal transition due to EB irradiation and that a onedimensional (1D) peanut-shaped connection is formed, where $\mathrm{C}_{60}$ molecules are linked by $s p^{2}$-like connections instead of $s p^{3}$-like connections, ${ }^{3}$ and intensive studies on electron transport in the $\mathrm{C}_{60}$ polymer have been carried out. ${ }^{4}$ First-principles calculations on the electronic structure of three-dimensional (3D) $\mathrm{C}_{60}$ polymers have revealed that $1 \mathrm{D}$ peanut-shaped $\mathrm{C}_{60}$ polymers are insulators, and electrons are conducted across the $1 \mathrm{D}$ polymers via $[2+2]$ four-membered rings between the two peanut-shaped $\mathrm{C}_{60}$ polymers in the $3 \mathrm{D}$ hexagonal structure ${ }^{5,6}$ which is a completely different configuration from the face-centered cubic structure of $\mathrm{C}_{60}$ bulk.

Nakaya et al. recently revealed that the chemical linkages between $\mathrm{C}_{60}$ molecules created by EB irradiation are thermally more stable than the $[2+2]$ four-membered ring because the polymers are stable under thermal annealing at $220^{\circ} \mathrm{C}$, which is higher than the decomposition temperature of the [2+2] four-membered rings, and they claimed that a peanut-shaped fullerene contributes to the metallic characteristics of EB irradiated films. ${ }^{7}$ They also demonstrated by scanning-tunneling spectroscopy (STS) that the characteristic peak of the spectra of the $\mathrm{C}_{60}$ molecule disappears at its polymerized center after EB irradiation. Moreover, their scanning tunneling microscopy (STM) images demonstrated that the $\mathrm{C}_{60}$ polymers retain a rhombohedral structure at the initial stage of EB irradiation; however, this result does not agree with the hexagonal structure derived by Onoe et al. using first-principles calculations. ${ }^{5,6}$ Thus, the physics underlying the generation of conductivity in EB irradiated $\mathrm{C}_{60}$ films remains unclear.

In this paper, an atomic configuration for the EB irradiated $\mathrm{C}_{60}$ film is proposed by using first-principles calculations. We examine what effect the bond configuration at the molecule junctions has on the electronic structure and electron-transport properties in order to interpret the generation of conductivity in EB irradiated $\mathrm{C}_{60}$ films. Our findings are that the rhombohedral $\mathrm{C}_{60}$ polymers consisting of [2+2] four-membered rings and $s p^{3}$-like interlayer connections create a semiconductor with a narrow band gap, and the band gap vanishes when the $s p^{2}$-like interlayer connection is formed. We found significant differences between the conduction spectra of dimers bonded by $s p^{3}$-like and $s p^{2}$-like connections; the conductance of the $s p^{2}$-like bonded dimer was higher than that of the $s p^{3}$-like bonded dimer below the Fermi level, and the peak of the conductance spectrum caused by the $t_{u 1}$ orbitals of the $s p^{3}$-like bonded dimer was clear while that of the $s p^{2}$-like bonded dimer was obscure.

All calculations are performed within the framework of density functional theory ${ }^{8}$ using the real-space finitedifference approach, ${ }^{9}$ which enables us to determine the self-consistent electronic ground state with a high degree of accuracy by using a time-saving double-grid technique. ${ }^{10,11}$ The norm-conserving pseudopotentials ${ }^{12}$ of Troullier and Martins $^{13}$ are used to describe electron-ion interaction, and exchange-correlation effects are treated with the local-density approximation. ${ }^{14}$

\section{ELECTRONIC STRUCTURE OF C 60 POLYMERS}

$\mathrm{C}_{60}$ polymers form a triangular lattice with $[2+2]$ fourmembered rings in each polymerized layer, and the layers 

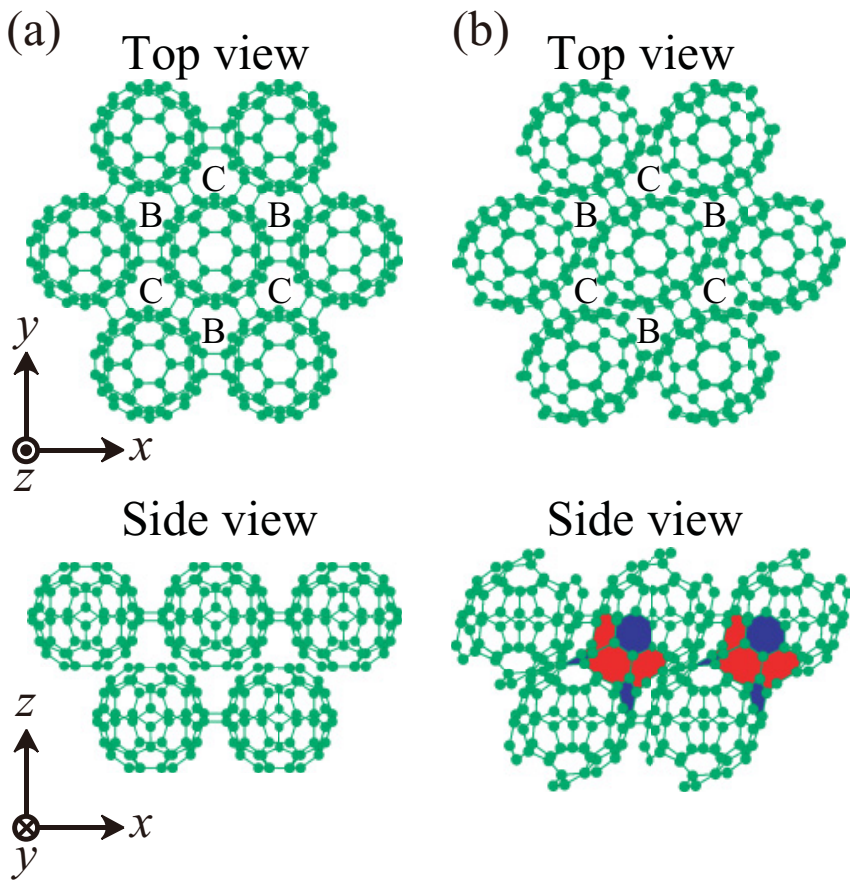

FIG. 1. (Color online) Geometric structure of 3D $\mathrm{C}_{60}$ polymer phases. (a) Polymer bonded by only $[2+2]$ four-membered rings and dumbbell-shaped connections and (b) polymer in which one of three dumbbell-shaped connections deforms to the peanut-shaped connection. The shaded segments in (b) represent the hexagons (blue) and heptagons (red) of the peanut-shaped connection. $B$ and $C$ in the top views correspond to the positions of $\mathrm{C}_{60}$ molecules in the upper and lower layers.

are stacked along a direction perpendicular to the layers in a rhombohedral symmetry. ${ }^{2}$ When an $A B C$-stacking structure ${ }^{15}$ is formed, the layers are bonded by $s p^{3}$-like connections, in which the hexagons on neighboring $\mathrm{C}_{60}$ molecules are brought face to face. When the $\mathrm{C}-\mathrm{C}$ bonds sheared by a hexagon and a pentagon on the facing hexagons are distorted, the layers are linked by an $s p^{2}$-like connection consisting of hexagons and heptagons. From now on, we will refer to $s p^{3}$ like interlayer connections as dumbbell shaped and $s p^{2}$-like interlayer connections as peanut shaped. The peanut-shaped connection is much stronger than the [2+2] four-membered ring because the energy to form it is smaller than that for the $[2+2]$ four-membered ring by $0.02 \mathrm{eV} /$ atom..${ }^{16}$ Therefore, the polymer can be reinforced by the peanut-shaped connection so that it can survive under thermal annealing. We first calculate the electronic structure of the $\mathrm{C}_{60}$ polymers.

Figure 1 shows two geometries of the models studied here. The $\mathrm{C}_{60}$ polymers in model (a) are bonded by the $[2+2]$ four-membered rings in the layer and linked by the dumbbell-shaped connections between the layers. In model (b), one of the dumbbell-shaped connections between the layers is deformed into the peanut-shaped connection. Since the metallic phase of the $\mathrm{C}_{60}$ polymer is surrounded by the other phase of the $\mathrm{C}_{60}$ 's in the initial stage of the $\mathrm{EB}$ irradiation according to the STM image, ${ }^{7}$ its lattice parameters are not expected to be fully relaxed. We assume lattice parameters $a=17.37$ bohrs, $c=46.30$ bohrs, and $\alpha=\pi / 3$, which correspond to those for the dumbbell-shaped interlayer
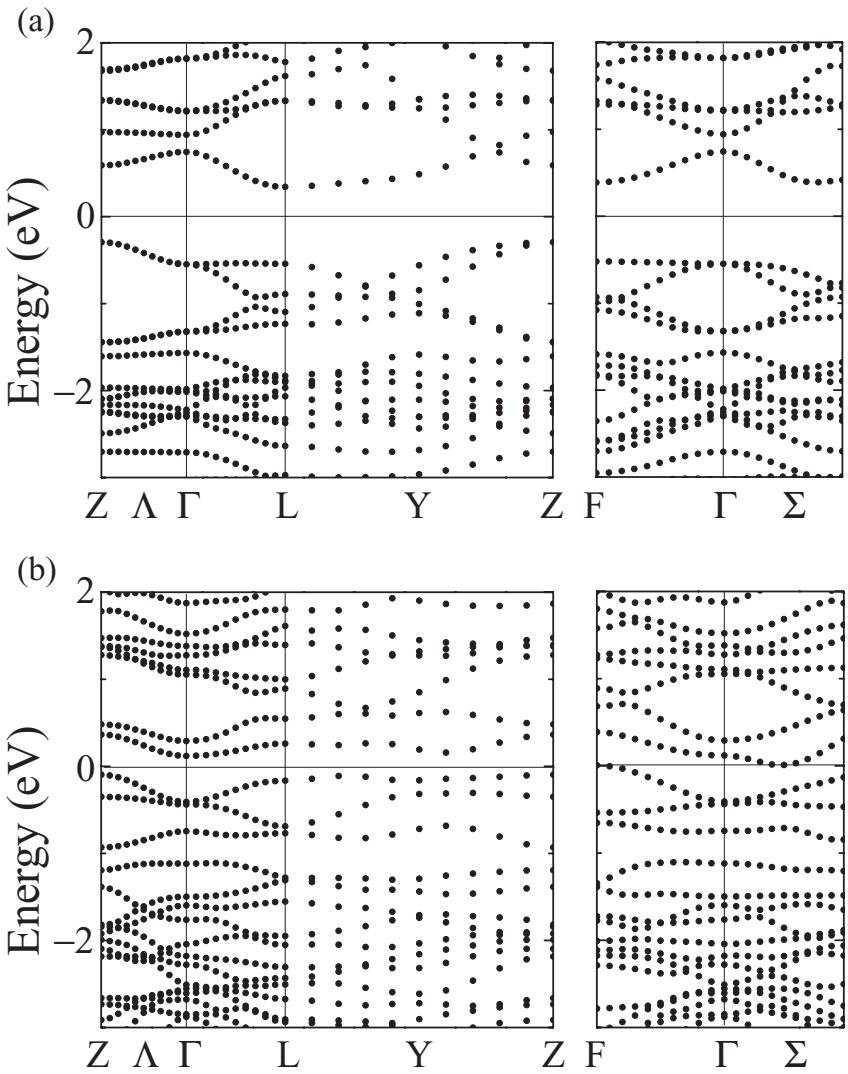

FIG. 2. Band structures of $3 \mathrm{D} \mathrm{C}_{60}$ polymers of models (a) and (b) in Fig. 1. Zero energy was chosen as the Fermi level.

connection obtained by x-ray diffraction pattern analysis, ${ }^{17}$ and employ these lattice parameters for both models (a) and (b) to compare the contributions of the dumbbell-shaped and peanut-shaped connections to the electronic structures. Note that the stacking structure of the polymer presented in this study is not deformed from that of the rhombohedral bulk phase and corresponds to the experimentally observed STM image, whereas the hexagonal polymers in Refs. 5 and 6 require drastic structural deformation. Integration in the Brillouin zone is carried out using 24-point sampling, and structural optimization for the atomic geometries is implemented for both models until the remaining forces for each atom are less than $36 \mathrm{meV} /$ bohr. The calculated electronic band structures are depicted in Fig. 2. The energy band gap is reduced by forming peanut-shaped connections; the peanut-shaped polymer exhibits metallic behavior while model (a) is a semiconductor with a fundamental band gap of $\sim 0.6 \mathrm{eV}$. The formation energy of model (b) is larger than that of model (a) by $0.25 \mathrm{eV} /$ atom although the formation energy of an isolated peanut-shaped dimer is smaller than that of a dumbbell-shaped dimer by $0.04 \mathrm{eV} /$ atom. Thus, the peanut-shaped connections are not widely generated but $\mathrm{C}_{60}$ molecules form small polymers with peanut-shaped connections in the film after EB irradiation.

\section{TRANSPORT PROPERTIES OF $\mathrm{C}_{60}$ DIMERS}

The STS spectra, which have been employed to interpret the transport properties of molecules, show peaks which are 
distinctly associated with the electronic structure of molecules. ${ }^{18}$ However, to replicate the STS spectrum of a $\mathrm{C}_{60}$ polymer consisting of more than $20 \mathrm{C}_{60}$ molecules in a $\mathrm{C}_{60}$ film, a system including a large number of atoms is required; this is not an easy task with the present computational resources. Moreover, the number of peaks measured for the considered bias-voltage range has been smaller than the number of electronic states in the molecules within the corresponding energy window in some cases. ${ }^{19}$ This fact implies that some states of molecules do not contribute to electron transport and that a simple interpretation of transport properties in terms of only the density of states (DOS) is insufficient. In addition, it is well known that the junctions between $\mathrm{C}_{60}$ molecules are bottlenecks for electron transport ${ }^{20-22}$ and the energy gap between the highest occupied molecular orbital (HOMO) and the lowest unoccupied molecular orbital (LUMO) decreases after polymerization. ${ }^{5,6,16,23}$ One of the present authors (S.T.) examined variations in the local density of states (LDOS) along the $1 \mathrm{D} \mathrm{C}_{60}$ polymer axis and demonstrated that the energy gap of the LDOS at the molecule junction is larger than that in $\mathrm{C}_{60}$ molecules. ${ }^{16}$ The $s p^{2}$-like connections of the peanut-shaped polymer might enhance electron transport because $\pi$ electrons are major carriers in metallic carbon nanotubes. Thus, it is of considerable interest to examine how the difference in the bond network between fullerenes contributes to electron transport through fullerenes.

We explore the contribution of the peanut-shaped connection to electron-transport properties. Figure 3 shows the computational model, where a $\mathrm{C}_{60}$ dimer is sandwiched between electrodes. Since the lattice-constant mismatch between the $\mathrm{C}_{60}$ polymers and the electrodes gives rise to further complex discussions on the transport properties, we employ simplified models to focus on the difference in the contributions to the transport properties between the dumbbell-shaped and peanut-shaped connections. To determine the optimized atomic coordinates and Kohn-Sham effective potential, we use a conventional supercell under a periodic boundary condition in all directions with a real-space grid spacing of $\sim 0.32$ bohrs; the dimensions of the supercell are $L_{x}=37.87$ bohrs, $L_{y}=$ 37.49 bohrs, and $L_{z}=71.15$ bohrs, where $L_{x}$ and $L_{y}$ are the lateral lengths of the supercell in the $x$ and $y$ directions parallel to the electrode surfaces, respectively, and $L_{z}$ is the length in the $z$ direction. Structural optimizations are implemented in advance for the isolated peanut-shaped dimer and it is then placed between the electrodes, where the three topmost surface atomic layers are atomistic $\mathrm{Al}(111)$ and the rest are aluminum jellium. The dimer is aligned at the hexagonal-close-packed hollow site on the (111) surface facing a hexagon, which is the most stable configuration for a $\mathrm{C}_{60}$ molecule on a face-centered-cubic (111) surface according to first-principles calculations. ${ }^{24}$ The distance between the surface atomic layers on the left and right electrodes is set at 35.81 bohrs so that the distance between the edge atoms of the dimer and the surface atomic layer of the electrodes corresponds to that reported by first-principles calculations. The dimer is relaxed between the electrodes. The atomic geometries of 36 carbon atoms for the dumbbell-shaped dimer at the molecule junction are modified from the above-mentioned structure and structural optimization is also implemented. (a)

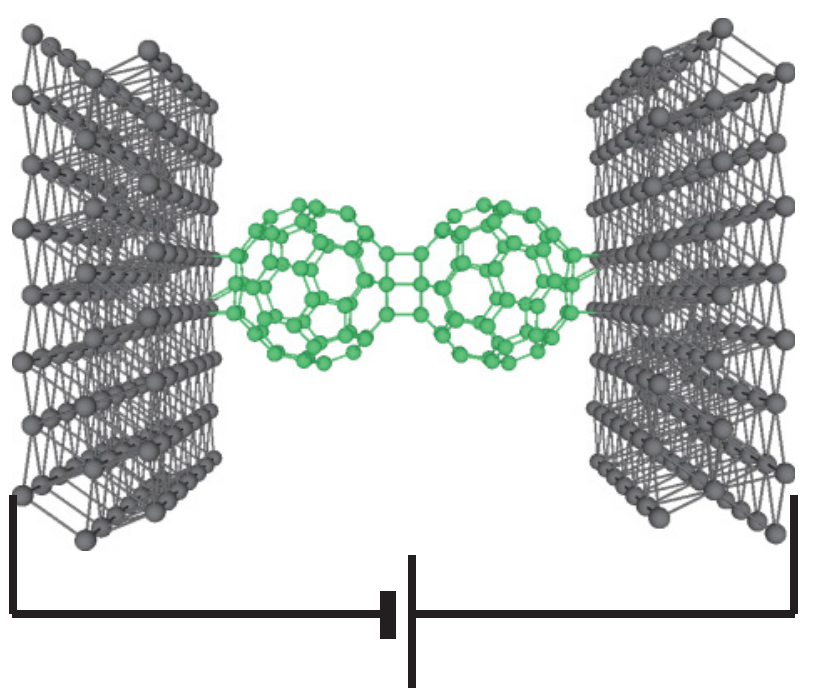

(b)

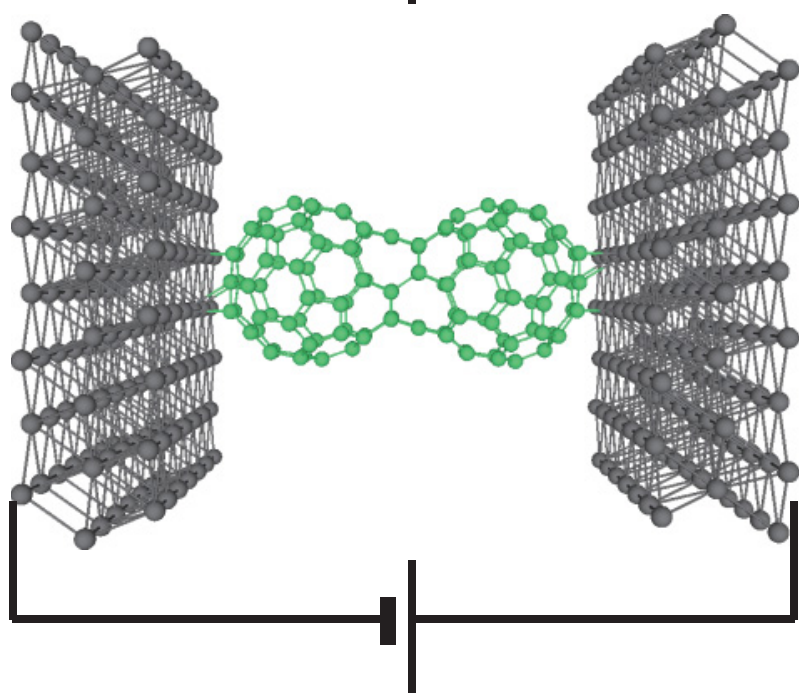

FIG. 3. (Color online) Computational model where $\mathrm{C}_{60}$ dimer is suspended between $\mathrm{Al}(111)$ electrodes. (a) Dumbbell-shaped dimer and (b) peanut-shaped dimer.

We take a grid spacing of $\sim 0.47$ a.u for the electrontransport calculations. We ensured that the decreased grid spacing and the enlarged supercell would not significantly affect our results. The scattering wave functions of the electrons propagating from the electrodes are determined by using the method of overbridging boundary matching. ${ }^{25-27}$ The retarded self-energy matrices for aluminum jellium are employed to include the rest of the semi-infinite electrodes. Since the DOS of aluminum is similar to that of free electrons, unfavorable effects from the DOS of the electrodes on the conductance spectra can be eliminated. We first calculate the Kohn-Sham effective potential using the supercell employed in structural optimization and then compute the scattering wave functions obtained non-self-consistently. It has been reported that this procedure is just as accurate in the linearresponse regime but significantly more efficient than performing computations self-consistently on a scattering-wave basis. ${ }^{28}$ The conductance of the dimers is described by the Landauer-Büttiker formula $G=\operatorname{Tr}\left(\mathbf{T}^{\dagger} \mathbf{T}\right) G_{0},{ }^{29}$ where $\mathbf{T}$ is the transmission-coefficient matrix. 


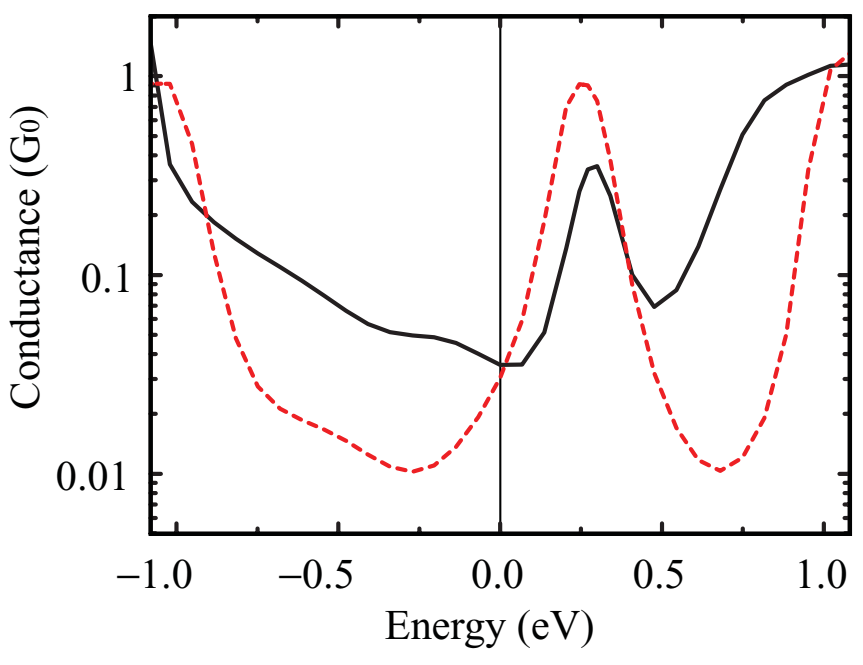

FIG. 4. (Color online) Conductance spectra as function of energy of incident electrons. The dashed curve represents the conductance of the dumbbell-shaped dimer and the solid curve that of the peanutshaped dimer. Zero energy was chosen as the Fermi level.

Figure 4 plots the conductance spectra of the dumbbellshaped and peanut-shaped dimers as a function of the energy of incident electrons. The magnitude of conductance at the Fermi level and the conduction spectra as a function of the energy of the incident electrons for the dumbbell-shaped dimer are in agreement with those reported in another theoretical study. ${ }^{22}$ Since the dimers are not connected by the $[2+2]$ four-membered rings in the direction parallel to the electrode and the $1 \mathrm{D} \mathrm{C}_{60}$ polymers act as an insulator, the conductance of the dimers is low around the Fermi level. The peak at $E_{F}+0.25 \mathrm{eV}$ is attributed to the triply degenerate LUMO $\left(t_{u 1}\right)$ of the $\mathrm{C}_{60}$ molecule, where $E_{F}$ is the Fermi level. The conductance of the peanut-shaped dimer is higher than that of the dumbbell-shaped dimer below the Fermi level, and the peak of the conductance spectrum induced by the $t_{u 1}$ orbitals of the dumbbell-shaped dimer is clearer than that of the peanut-shaped dimer. The strong peak of the conductance spectrum of the dumbbell-shaped dimer corresponds to the contribution of the resonant tunneling effect through the triply degenerate $t_{u 1}$ orbitals. On the other hand, the characteristic feature of the resonant tunneling is insignificant when the peanut-shaped connections are formed.

To examine the contribution of the peanut-shaped connection to the electron-transport properties in more detail, Fig. 5 shows the LDOSs of the dimers, which have been plotted by integrating them along the plane parallel to the electrode surface, $\rho(z, E)=\int|\psi(\boldsymbol{r}, E)|^{2} d \boldsymbol{r}_{\|}$, where $\boldsymbol{r}=(x, y, z), \psi$ is the wave function, and $E$ is the energy of the states. The LDOS at $E_{F}-0.2 \mathrm{eV}$ around the molecule junction of the peanut-shaped dimer, which is indicated by the black arrows in Fig. 5, is larger than that of the dumbbell-shaped dimer. In addition, as indicated by the red arrows in Fig. 5, the energetically discrete and spatially localized $t_{u 1}$ orbitals of the $\mathrm{C}_{60}$ molecules are deformed to energetically and spatially broadened states by the formation of the peanut-shaped $s p^{2}$ like connections at the molecule junction: the significant peaks of the LDOS due to the triply degenerate $t_{u 1}$ orbitals are low, and the low-density regions at the molecule junction become small or disappear. The incident electrons from the electrode can easily penetrate into the peanut-shaped dimer via energetically broadened states, and thus the conductance spectrum in Fig. 4 becomes obscure. These results imply that the formation of the peanut-shaped dimer contributes to the metallic properties of the EB irradiated $\mathrm{C}_{60}$ film by increasing the electron hopping between $\mathrm{C}_{60}$ molecules. (a)

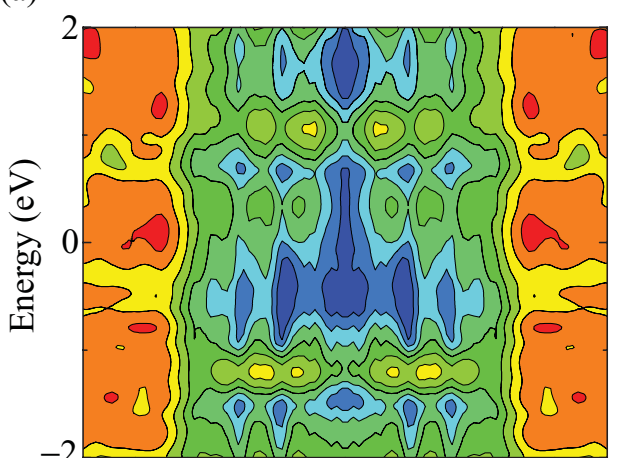

(b)

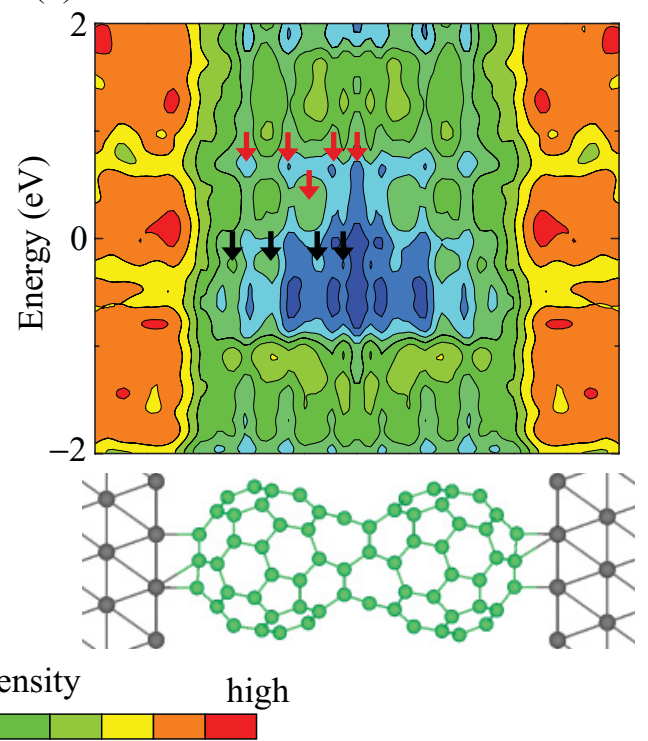

FIG. 5. (Color) Distributions of LDOS integrated on plane parallel to dimer as functions of relative energy from Fermi level. (a) Ddumbbell-shaped dimer and (b) peanut-shaped dimer. Zero energy is chosen as the Fermi level. Each contour represents twice or half the density of the adjacent contour lines, and the lowest contour is $2.56 \times 10^{-4} \mathrm{e} / \mathrm{eV} / \mathrm{bohr}$. The atomic configurations below the graph are visual guides. 


\section{SUMMARY}

The atomic configuration of an EB irradiated $\mathrm{C}_{60}$ film has been proposed to explain the conductivity in the $\mathrm{C}_{60}$ film through first-principles calculations. Although the polymer composed of $[2+2]$ four-membered rings and dumbbellshaped interlayer connections is a semiconductor with a narrow band gap, the polymer changes to exhibit metallic characteristics by forming peanut-shaped interlayer connections. Calculations of the electron transport for the dumbbell-shaped and peanut-shaped dimers revealed higher conductance of the peanut-shaped dimer below the Fermi level than that of the dumbbell-shaped dimer. The low peak of the conductance spectrum induced by the $t_{u 1}$ orbitals of the peanut-shaped dimer is related to the energetically broadened LDOS at the molecule junction caused by the formation of the $s p^{2}$-bonded peanut-shaped connections and the metallic property of the EB irradiated $\mathrm{C}_{60}$ film. A rhombohedral film consisting of $[2+$ 2] four-membered rings with dumbbell-shaped and peanutshaped connections is a possible structure for the metallic $\mathrm{C}_{60}$ polymer observed by STM in the initial stage of EB irradiation, since the present structure could be formed without significant structural deformation from face-centered-cubic $\mathrm{C}_{60}$ bulk and agrees with the rhombohedral structure in the STM image.
Although our computational models are limited to simplified $\mathrm{C}_{60}$ polymers owing to our limited computational resources, large-scale first-principles calculations for $\mathrm{C}_{60}$ polymers may help to validate the correlation between the STS spectra and the present atomic configuration of the polymers and will be carried out in the future.

\section{ACKNOWLEDGMENTS}

The authors would like to thank Kikuji Hirose and Yoshitada Morikawa of Osaka University and Yoshiyuki Egami of Nagasaki University for fruitful discussion. This research was partially supported by Strategic Japanese-German Cooperative Program from Japan Science and Technology Agency and Deutsche Forschungsgemeinschaft, and by a Grant-in-Aid for Scientific Research on Innovative Areas (Grant No. 22104007) from the Ministry of Education, Culture, Sports, Science and Technology, Japan. The numerical calculation was carried out using the computer facilities of the Institute for Solid State Physics at the University of Tokyo, the Research Center for Computational Science at the National Institute of Natural Science, the Center for Computational Sciences at the University of Tsukuba, and the Information Synergy Center at Tohoku University.

\footnotetext{
${ }^{1}$ Fullerene Polymers and Fullerene Polymer Composites, edited by P. C. Eklund and A. M. Rao (Springer, Berlin, 2000), and references therein.

${ }^{2}$ S. Okada and A. Oshiyama, Phys. Rev. B 68, 235402 (2003).

${ }^{3}$ J. Onoe, T. Nakayama, M. Aono, and T. Hara, Appl. Phys. Lett. 82, 595 (2003).

${ }^{4}$ See, e.g., J. Onoe, A. Takashima, and Y. Toda, Appl. Phys. Lett. 97, 241911 (2010).

${ }^{5}$ S. Ueda, K. Ohno, Y. Noguchi, S. Ishii, and J. Onoe, J. Phys. Chem. B 110, 22374 (2006).

${ }^{6}$ J. Onoe, T. Ito, S. I. Kimura, K. Ohno, Y. Noguchi, and S. Ueda, Phys. Rev. B 75, 233410 (2007).

${ }^{7}$ M. Nakaya, M. Aono, and T. Nakayama, Carbon 49, 1829 (2011).

${ }^{8}$ P. Hohenberg and W. Kohn, Phys. Rev. 136, B864 (1964).

${ }^{9}$ J. R. Chelikowsky, N. Troullier, and Y. Saad, Phys. Rev. Lett. 72, 1240 (1994); J. R. Chelikowsky, N. Troullier, K. Wu, and Y. Saad, Phys. Rev. B 50, 11355 (1994).

${ }^{10}$ K. Hirose, T. Ono, Y. Fujimoto, and S. Tsukamoto, First Principles Calculations in Real-Space Formalism, Electronic Configurations and Transport Properties of Nanostructures (Imperial College Press, London, 2005).

${ }^{11}$ T. Ono and K. Hirose, Phys. Rev. Lett. 82, 5016 (1999); Phys. Rev. B 72, 085115 (2005); T. Ono, M. Heide, N. Atodiresei, P. Baumeister, S. Tsukamoto, and S. Blügel, ibid. 82, 205115 (2010).

${ }^{12} \mathrm{We}$ used the norm-conserving pseudopotentials NCPS97 constructed by K. Kobayashi using the procedure proposed in Ref. 13. See K. Kobayashi, Comput. Mater. Sci. 14, 72 (1999).

${ }^{13}$ N. Troullier and J. L. Martins, Phys. Rev. B 43, 1993 (1991).
}

${ }^{14}$ J. P. Perdew and A. Zunger, Phys. Rev. B 23, 5048 (1981).

${ }^{15}$ X. Chen, S. Yamanaka, K. Sako, Y. Inoue, and M. Yasukawa, Chem. Phys. Lett. 356, 291 (2002).

${ }^{16}$ S. Tsukamoto and T. Nakayama, J. Chem. Phys. 122, 074702 (2005).

${ }^{17}$ M. Núñez-Regueiro, L. Marques, J.-L. Hodeau, O. Béthoux, and M. Perroux, Phys. Rev. Lett. 74, 278 (1995).

${ }^{18}$ J. Tersoff and D. R. Hamann, Phys. Rev. B 31, 805 (1985).

${ }^{19}$ H. Hövel, B. Grimm, M. Bödecker, K. Fieger, and B. Reihl, Surf. Sci. 463, L603 (2000); H. Hövel and I. Barke, Prog. Surf. Sci. 81, 53 (2006); M. De Menech, U. Saalmann, and M. E. Garcia, New J. Phys. 9, 340 (2007).

${ }^{20}$ M. Otani, T. Ono, and K. Hirose, Phys. Rev. B 69, 121408(R) (2004).

${ }^{21}$ T. Ono and K. Hirose, Phys. Rev. Lett. 98, 026804 (2007).

${ }^{22}$ G. Schull, T. Frederiksen, M. Brandbyge, and R. Berndt, Phys. Rev. Lett. 103, 206803 (2009).

${ }^{23}$ H. Nakayama, T. Ono, H. Goto, and K. Hirose, Sci. Technol. Adv. Mater. 8, 196 (2007).

${ }^{24}$ L.-L. Wang and H.-P. Cheng, Phys. Rev. B 69, 165417 (2004).

${ }^{25}$ Y. Fujimoto and K. Hirose, Phys. Rev. B 67, 195315 (2003).

${ }^{26}$ L. Kong, M. L. Tiago, and J. R. Chelikowsky, Phys. Rev. B 73, 195118 (2006)

${ }^{27}$ Y. Egami, K. Hirose, and T. Ono, Phys. Rev. E 82, 056706 (2010).

${ }^{28}$ L. Kong, J. R. Chelikowsky, J. B. Neaton, and S. G. Louie, Phys. Rev. B 76, 235422 (2007).

${ }^{29}$ M. Büttiker, Y. Imry, R. Landauer, and S. Pinhas, Phys. Rev. B 31, 6207 (1985). 